\title{
Target Acquisition performance: effects of target aspect angle, dynamic imaging and signal processing
}

\author{
Jaap A. Beintema ${ }^{\mathrm{a}}$, Piet Bijl ${ }^{\mathrm{a}}$, Maarten A. Hogervorst ${ }^{\mathrm{a}}$ and Judith Dijk ${ }^{\mathrm{b}}$ \\ ${ }^{a}$ TNO Defense, Security \& Safety: Human Factors, P.O. Box 23, Soesterberg, The Netherlands \\ ${ }^{\mathrm{b}}$ TNO Defense, Security \& Safety: Observation Systems, The Hague, The Netherlands \\ Phone: +31346356368, fax: +31346353977 \\ E-mail: jaap.beintema@tno.nl, piet.bijl@tno.nl,maarten.hogervorst@tno.nl,judith.dijk@tno.nl
}

\begin{abstract}
In an extensive Target Acquisition (TA) performance study, we recorded static and dynamic imagery of a set of military and civilian two-handheld objects at a range of distances and aspect angles with an under-sampled uncooled thermal imager. Next, we applied signal processing techniques including DSR (Dynamic Super Resolution) and LACE (Local Adaptive Contrast Enhancement) to the imagery. In a perception experiment, we determined identification (ID) and threat/non-threat discrimination performance as a function of target range for a variety of conditions. The experiment was performed to validate and extend current TA models. In addition, range predictions were performed with two TA models: the TOD model and NVThermIP. The results of the study are: i) target orientation has a strong effect on performance, ii) the effect of target orientation is well predicted by the two TA models, iii) absolute identification range is close the range predicted with the two models using the recommended criteria for two-handheld objects, iv) there was no positive effect of sensor motion on performance, and this was against the expectations based on earlier studies, v) the benefit of DSR was smaller than expected on the basis of the model predictions, and vi) performance with LACE was similar to performance on an image optimized manually, indicating that LACE can be used to optimize the contrast automatically. The relatively poor results with motion and DSR are probably due to motion smear induced by a higher camera speed than used in earlier studies. Camera motion magnitude and smear are not yet implemented in TA models.
\end{abstract}

Keywords: Target Acquisition, dynamic imaging, sensor performance, validation, image enhancement, super resolution, local adaptive contrast enhancement, TOD, NVThermIP

\section{INTRODUCTION}

For decades, the focus of Target Acquisition performance measurement and modeling has been on static imaging of military vehicles at long range using a high-end scanning or staring thermal imager ${ }^{1,2,3,4,5,6}$. In recent years an important shift of interest has taken place to other scenes with different objects of interest and different types of sensors. For instance, terrorists using small arms form a serious threat in urban and littoral environments. To be able to identify these as a threat, small weapons such as guns, knives, machine guns or even an RPG need to be distinguished from everyday civilian objects such as a cell phone, a video camera, a broom or a pipe. Operating ranges in the urban environment are typically much shorter than in the traditional battlefield and the dynamics in the scene or the sensor have become much more important. At the same time hand-held, uncooled thermal imagers such as micro-bolometers have entered the market. Their price is attractive but the other side of the coin of course is a relatively poor resolution and low thermal sensitivity. Other sensor developments include different spectral bands such as the Mid Wave IR region and a fast extension of the number of cameras in the visible region. Finally, signal processing techniques such as DSR (Dynamic Super Resolution), SBNUC (Scene-Based Non-Uniformity Correction) and LACE ${ }^{34}$ (Local Adaptive Contrast Enhancement) that process the sensor image in real-time become available now. The impact of motion and dynamic techniques such as DSR and SBNUC is highest on the low-cost thermal imagers because these are the most undersampled $^{7,8}$ and have the highest noise levels. 
TA models ${ }^{9-16}$ such as the TOD model, NVThermIP and TRM3 need to be extended and validated for these largely different target sets, sensor properties, image dynamics and processing techniques, but currently the amount of empirical data is limited and often only addresses one of the above-mentioned differences at the same time. For instance, a large effort of the US research laboratory is directed towards extension of the current models for different target sets such as small arms $^{17,18}$, clothing $^{19}$, paramilitary and civilian vehicles ${ }^{20}$, small boats ${ }^{21}$, or even faces ${ }^{22}$ in different spectral regions. A recent overview of the results is given by Driggers et al. $^{23}$. However, the imagery used in the experiments are static and recorded at a single short range. Sensor sampling and target range are represented by different amounts of blur using an empirical relationship that is also implemented in the NVThermIP model (the 'MTFsqueeze' approach ${ }^{24}$ ). Thus strictly these data may not be used as a validation of NVThermIP. Effects of motion and DSR on under-sampled uncooled thermal imagery are studied in both laboratory and field ${ }^{25,26,27}$, but most are on the TOD triangle laboratory test pattern and not on realistic targets. Recently, one study has been reported on IR system field performance with super-resolution ${ }^{28}$, using a tank target set.

In the present study we take all aspects together and collect a set of present-day relevant TA data for model extension and validation purposes. With an uncooled WFOV micro-bolometer thermal imager we recorded static and dynamic IR imagery of five military and five civilian two-handheld objects at a range of distances and aspect angles and several types of signal processing techniques including DSR, LACE and combinations to the imagery. In a perception experiment, we determined identification and threat/non-threat discrimination performance as a function of target range and aspect angle for still, static, dynamic and enhanced imagery. Specific applications of the data set include:

- $\quad$ validation of earlier TA data reported for two-handheld targets ${ }^{23}: V_{50}$ for NVthermIP and $M_{75}$ for the TOD model $^{15}$, and slope of the probability vs range relationship.

- $\quad$ validation of the model assumption with respect to target aspect angle: is the target-square root area as used in current TA models indeed a good predictor for small elongated weapons?

- Extension of the current TA models for motion video, which has not yet been modeled.

- Validation and/or extension of the current TA models for DSR, LACE and combinations.

- validation of the TOD test method with respect to i) dynamic imaging and ii) a range of signal processing techniques $^{25,26,27}$ : does the TOD method correctly predict the effect of motion, DSR, LACE and combinations of these on the acquisition of real targets?

This paper is organized as follows. In Chapter 2, some examples of dynamic signal processing techniques are given. The experimental method is described in Chapter 3. The results of the experiments and model predictions are given in Chapter 4 and discussed in Chapter 5. Conclusions are given in Chapter 6.

\section{SIGNAL PROCESSING: STATIC AND DYNAMIC TECHNIQUES}

Contrast enhancement and luminance conversions to optimally exploit the monitor's luminance profile are signal processing techniques that can be applied to both static and dynamic imagery. Global contrast enhancement rescales a selected luminance range to the full monitor range. New techniques such as LACE allow mapping the whole luminance range to the full range of the monitor while enhancing local contrasts. In this study we apply LACE using a software package developed by TNO.

Example images of LACE applied to a static, dynamic and a DSR processed sequence (statLACE, dynLACE and dynDSR4+LACE) are shown in Figure 1. Also included for comparison are original sequence samples (stat, dyn and dynDSR4, respectively) after applying manual optimization of the luminance range for identification of the hand-held object. Note: without LACE only few features, among which the hand-held object are visible, whereas with LACE also other features, such as the folds in the curtain behind the object become visible.

Dynamic signal processing techniques such as DSR or SBNUC and many others have been widely reported in the literature ${ }^{29,30,31}$. These techniques make use of motion in the image to improve sampling resolution and/or to reduce 
temporal noise in an undersampled system. In this study we will use DSR processing from a comprehensive signal processing software package developed by $\mathrm{TNO}^{31}$. Figure 1 shows examples of applying DSR using DSR1, DSR2 and DSR4. DSR1 is a condition in which resolution has not been increased but temporal noise is integrated over a number of frames. DSR2 effectively results in a reduction of the detector pitch by a factor of 2 and a doubling of the sampling resolution. DSR4 reduces the pitch by $1 / 4$ and results in a fourfold sampling resolution. In all DSR conditions the super resolution calculations are preceded by SBNUC to avoid artefacts due to the fixed pattern noise.

Note that the optics and detector blur do not change with DSR. This means that imagery from an under-sampled imager are often well-sampled after application of DSR.

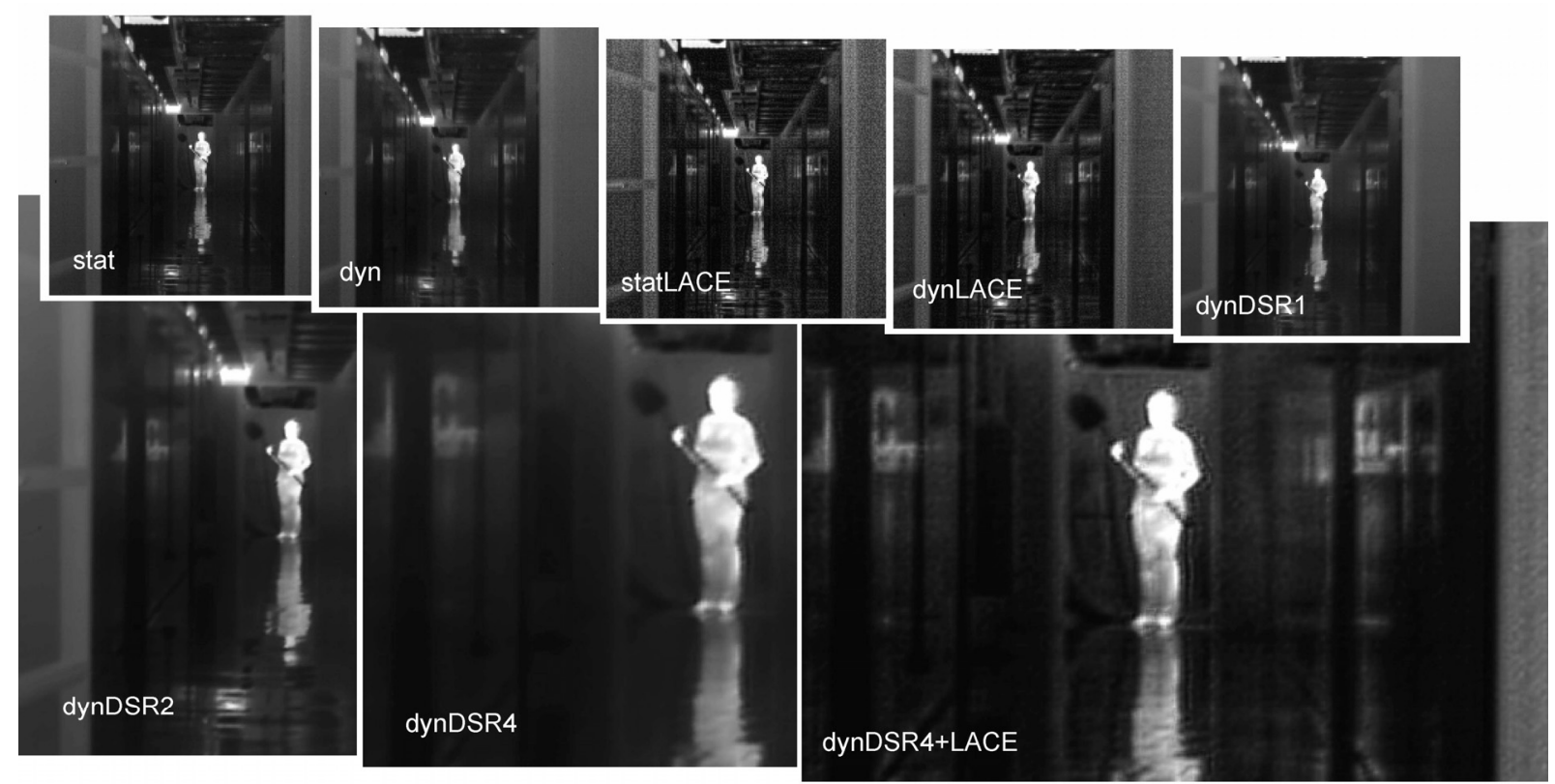

Figure 1. Example results of applying LACE (Local Adaptive Contrast Enhancement), DSR (Dynamic Super Resolution) and combined DSR with LACE on static and dynamic images. For details, see text.

\section{METHODS}

\section{1 Sensor}

We used a FLIR SC2000 undersampled, uncooled micro-bolometer sensor with a focal plane array of 320 by 240 pixels (see Figure 2). FOV is 24 by 18 degrees. The camera gives a calibrated output of the temperatures in the scene. Data was 14 bit digital recorded at a frame rate of $50 \mathrm{~Hz}$.

\subsection{Motion}

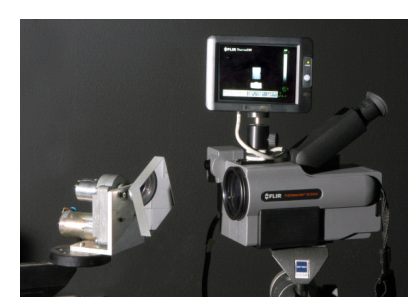

Figure 2. Camera and the rotating tilted mirror used to generate a dynamic image. 
A surface mirror was placed in front of the camera objective under an angle of approximately 45 degrees (see Figure 2) so that an image of the scene of interest was obtained. The mirror was mounted on the axis of an electric motor and slightly tilted with respect to the rotation axis in order to produce a circular motion of the image. Diameter and speed are set by the changing the tilt angle and rotation frequency.

\subsection{Image Collection}

For both static and dynamic recordings 100 frames were collected. For the dynamic recordings, the diameter of the circular motion was $0.34^{\circ}$ and the rotation frequency was $1.0 \mathrm{~Hz}$. This corresponds to a test pattern speed of approximately 0.57 pixel per frame.

Ten objects were selected (see Figure 3), consisting of 5 military weapons (Diemaco C7 rifle, Diemaco C8 rifle, LOAW automatic rifle, STEYR long-distance rifle, AT-4 RPG) and 5 civil objects (PVC pipe, axe, spade, metal stick, broom).
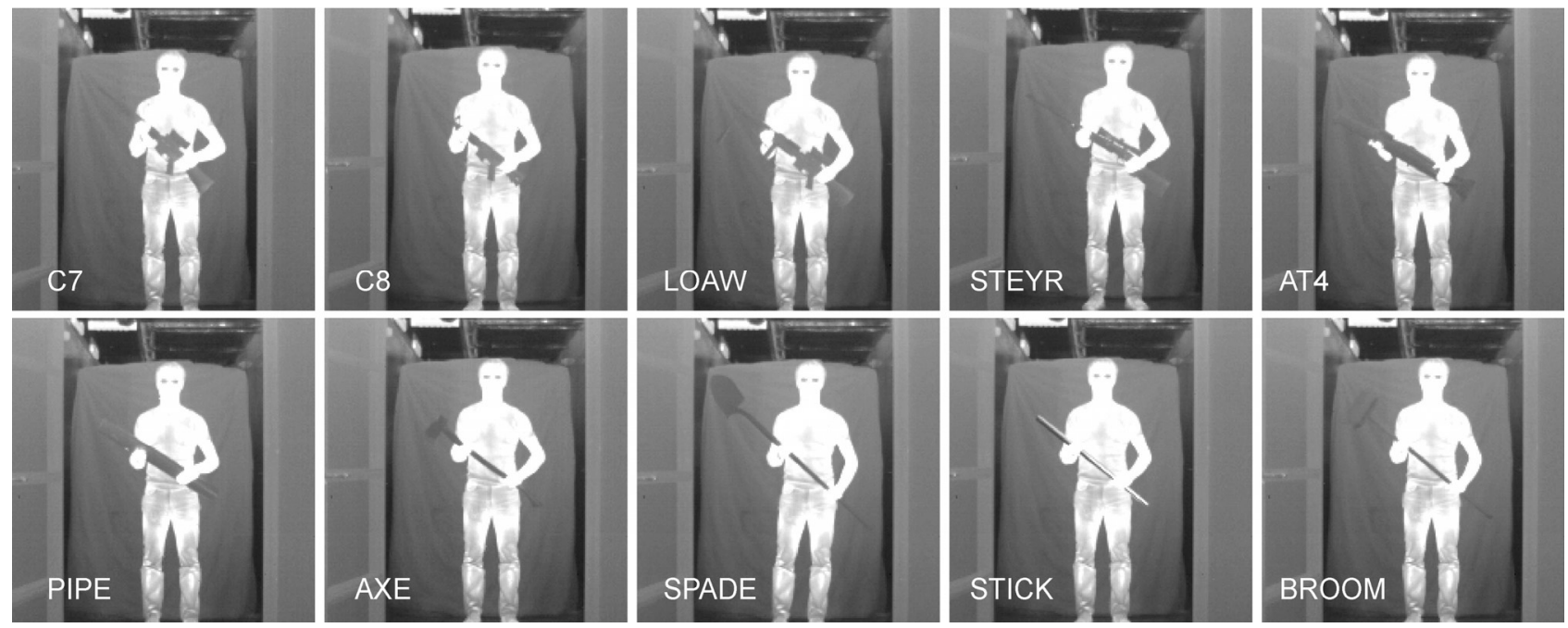

Figure 3. Example imagery of all two-handheld objects.

Targets were held diagonally at an angle of 45 degrees by one of the two actors (one male, one female). Five different aspect angles were used: 90 degrees left, 45 degrees left, zero degrees (side-view), 90 degrees right, and 45 degrees right (Figure 4).
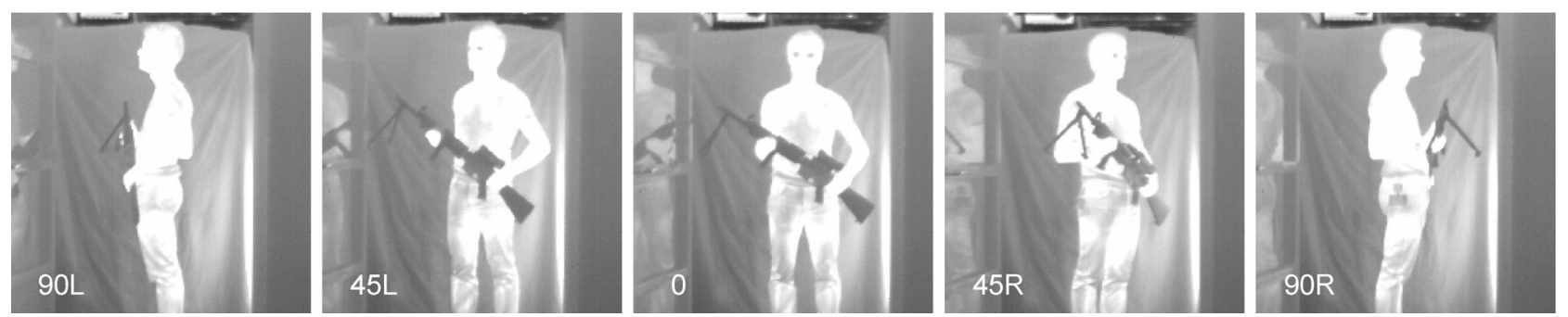

Figure 4. Example of a target at 5 different aspect angles.

Images were recorded in a long hallway at constant room temperature. Distances were 4.0, 5.04, 6.35, 8.0, 10.1, 12.7, $16.0,20.2,25.4,32.0,40.3$ and $50.8 \mathrm{~m}$ from the camera (Figure 5).Note that the background temperature decreases towards the middle of the alley. To prevent such confounding factor on range effects, the temperature of the immediate 
background was held constant by placing a curtain behind the object. The performance of LACE was put to test by creating large temperature differences using spotlights placed along the hallway, including a $2000 \mathrm{~W}$ lamp.
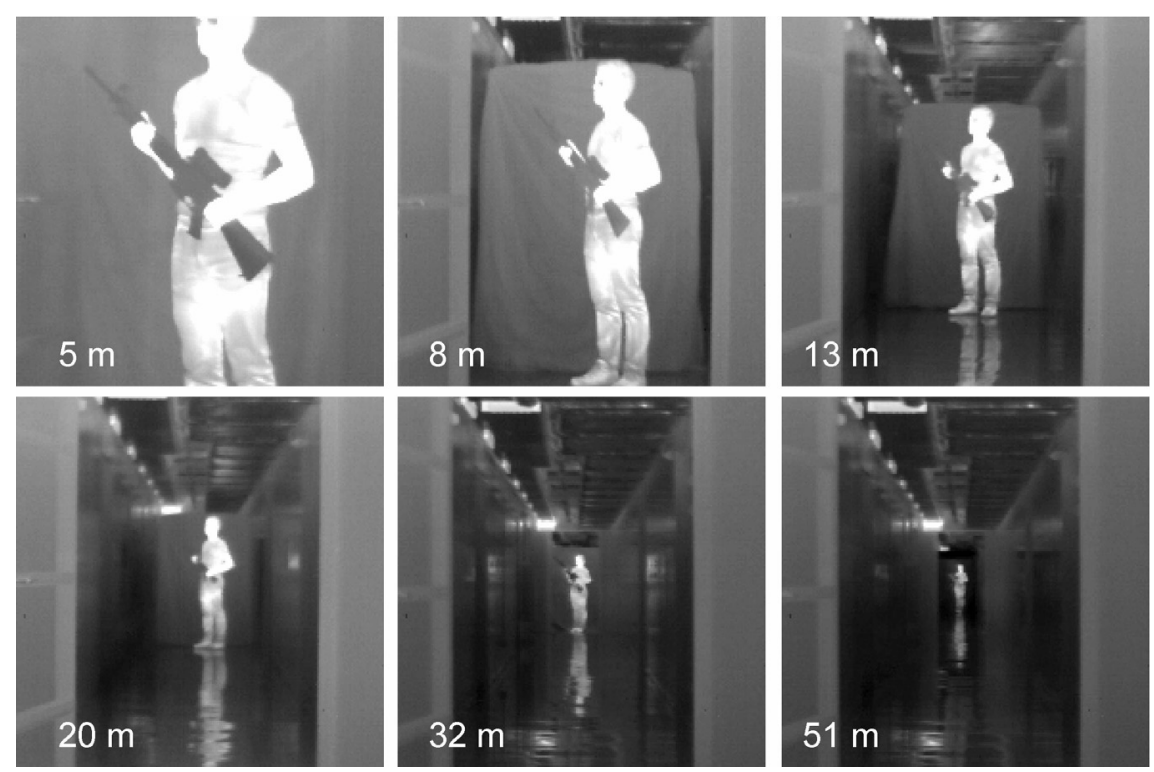

Figure 5. Example imagery of target at physically different distances.

\subsection{Signal processing}

Basically, 10 different conditions are used in this study. Four of these conditions are unprocessed: 'still' (which is a single frame taken from the static recordings), 'static' and 'dyn' (these are described in a previous section) and 'dyn0.5' which is the same as dynamic sequence but played back at half the recording speed.

In the other six conditions signal processing has been applied using the package developed at TNO (see Chapter 2). DSR was applied to the dynamic recordings only ('dynDSR1', 'dynDSR2' and 'dynDSR4'; see Chapter 2). LACE was applied to the static and dynamic recordings ('statLACE' and 'dynLACE') and in combination with DSR4 ('dynDSR4+LACE').

Except for the LACE conditions, the images were optimized manually by rescaling temperatures between 18.5 and 28.0 ${ }^{\circ} \mathrm{C}$ to the monitor luminance depth range, clipping the values outside this range. LACE automatically rescaled the luminance range.

\subsection{Experimental procedure of the perception test}

The experiments were carried out in a dimly lit room. Test patterns were shown on a 22-inch computer CRT set at low resolution (1024 by 768 pixels) to be sure that pattern size on the display is not a primary limiting factor. Subjects were free to choose the optimum distance from the display (most of the time distance was approximately $50 \mathrm{~cm}$ ). Subsequent frames were presented after each monitor refresh $(60 \mathrm{~Hz})$. The frame sequence was repeated to create continuous motion on the screen.

Each trial an image from the recorded set appeared on the screen which remained visible until the subject responded. Presentation order was balanced over observers. In a 10AFC (10-alternative forced-choice) paradigm, subjects were instructed to mouse-click one of the day-light images (see Figure 6) below the thermal image containing the target that best matched the presented one. There was no time limit. After the response, a next trial started. 

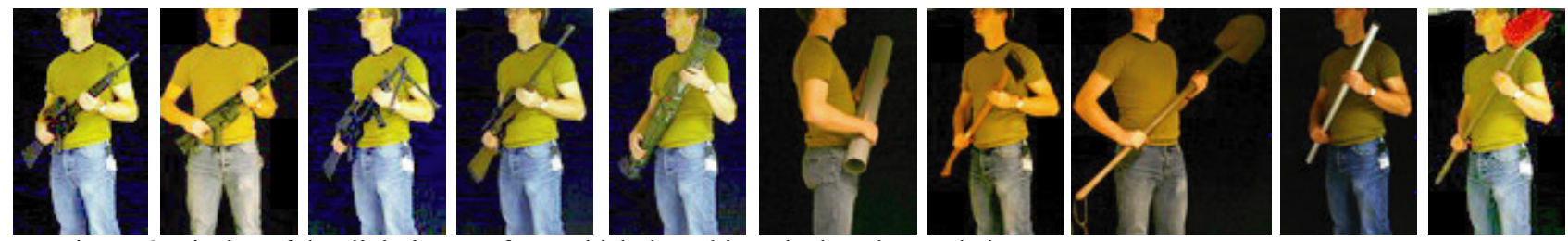

Figure 6. Display of day-light images from which the subjects had to choose their response.

All participants ( 7 students in experiment I and 8 different students in experiment II) had normal or corrected-to-normal vision. Subjects were not experienced with weapon identification. They received a brief introduction on the use of each weapon and practice trials without feedback using the same dataset (30 in Exp. I, 200 in Exp. II). Subjects took a rest after every 300 trials. Each experiment lasted about two hours.

\subsubsection{Experiment 1: target orientation and motion}

In the first experiment we investigated the effect of motion (static and dynamic) and aspect angle (5 angles). Each combination was presented once. In total, each subject received 1200 trials (10 objects x 12 distances x 5 target aspect angles x 2 motion conditions).

\subsubsection{Experiment 2: signal processing}

In this experiment we investigated the effect of image processing. Since DSR2 and DSR4 result in a higher resolution, they were shown 2 and 4 times larger on the display than the unprocessed imagery. Bijl et al. (2006) ${ }^{26}$ showed for TOD test targets that a simple $2 \mathrm{x}$ or $4 \mathrm{x}$ magnification of the original image did not improve performance. To check whether the motion speed influenced subjects performance, a dynamic condition was included in which the motion was played back at half the speed ('dyn0.5').

To limit the number of combinations, this experiment was carried out at one aspect angle only (45L and 45R, evenly balanced across subjects and distances). Each combination was presented only once. In total, each subject received 1000 trials (10 objects x 10 distances x 10 conditions), plus a repetition of the first 200 trials at the end of the run. These first 200 trials (practice trials) were omitted from the analysis.

\subsection{Observer response analysis}

Probability versus range data were fitted to a Weibull function:

(1) $P(r)=\gamma+(1-\gamma-\delta) *\left(1-e^{-(\alpha / x)^{\beta}}\right)$

having two free parameters (threshold $\alpha$ and slope $\beta$ ) and two fixed parameters (guess level $\gamma=0.1$ and lapse chance $\delta=0.05$ for identification, $\gamma=0.5$ and $\delta=0.01$ for threat/non-threat discrimination). The threshold $\alpha$ and slope $\beta$ were found by maximal likelihood estimation ${ }^{33}$. From the fit parameters, the $75 \%$ correct range $\left(\mathrm{r}_{75}\right)$ was determined.

\subsection{Model predictions}

\subsubsection{Characteristic target set parameters}

All currently accepted TA models (TOD model, NVThermIP and TRM3) take the average target square-root area (in m) as a measure of the effective target dimension and use the $\Delta$ TRSS (the squared sum of target-to-background and internal thermal contrast) in $\mathrm{K}$ as the characteristic contrast. 
Target effective dimension and thermal contrast were estimated on the original recorded imagery at shortest distance (4 $\mathrm{m})$ for each aspect angle. The characteristic parameters, averaged over 10 targets $( \pm \mathrm{SE})$, are given in Table 1 . In this study the internal thermal variation inside the targets is negligible compared to the target background contrast. Hence we use $\Delta \mathrm{T} \approx \Delta \mathrm{TRSS}$.

Note that both size and thermal contrast vary with target orientation, contrast and target size being slightly correlated $\left(\mathrm{R}^{2}=0.12\right.$, computed over $5 \times 10$ images $)$. This is explained by the decreased area of warm body that acts as background when the actor turns sideways.

Table 1: Characteristic parameters for the two-handheld target set

\begin{tabular}{|l|l|l|l|l|l|l|}
\hline & $90 \mathrm{~L}$ & $45 \mathrm{~L}$ & 0 & $45 \mathrm{R}$ & $90 \mathrm{R}$ & Average \\
\hline Size $(\mathrm{m})$ & $0.198 \pm .05$ & $0.260 \pm .05$ & $0.297 \pm .06$ & $0.249 \pm .05$ & $0.221 \pm .05$ & 0.245 \\
\hline$\Delta \mathrm{T}(\mathrm{K})$ & $1.31 \pm 0.3$ & $1.61 \pm 0.3$ & $1.63 \pm 0.3$ & $2.44 \pm 0.4$ & $0.61 \pm 0.2$ & 1.52 \\
\hline
\end{tabular}

Driggers et al. ${ }^{32}$ provide the recommended characteristic parameters for a similar two-handheld target set from an earlier study using a MWIR camera: characteristic size $=0.25 \mathrm{~m}$, characteristic $\Delta$ TRSS $=4.1 \mathrm{~K}$. These values are an average over all orientations used. Characteristic size is in excellent agreement with our average value of $0.245 \mathrm{~m}$. Their thermal contrast is a little higher. The difference can be due to a different experimental environment during recording (our recordings were made in a dark-painted basement) and/or due to the different spectral range of the sensor (LWIR vs. MWIR).

\subsubsection{Range prediction with the NVThermIP model}

The recommended $V_{50}$ is 16 for a two-handheld target set ${ }^{32}$. For the FLIR SC2000 camera (with an assumed fill factor of 1) we make range predictions with NVThermIP using the average characteristic parameters from Table 1. Scene temperature variation was set at $10 \mathrm{~K}$. The predicted $75 \%$ ID range for the static conditions with NVThermIP per aspect angle (90L, 45L, 0, 45R, 90R) are 10.25, 15.0, 17.2, 17.7 and $17.8 \mathrm{~m}$, with an average 75\% ID range of $13.6 \mathrm{~m}$.

\subsubsection{Range prediction with the TOD model}

The TOD range predictions for a two-handheld target set are also based on the effective size and $\Delta$ TRSS contrast as given in Table 1. For this set, the recommended TOD criterion (magnification factor $M_{75}$ ) can be deduced from the NVThermIP criteria as follows (Bijl et al. $\left.{ }^{15}\right)$ :

(2) $M_{75}=0.35 \cdot V_{75}=0.35 \cdot\left(V_{50} / 0.58\right)=9.7$

The predicted range $r_{75}$ (in $\mathrm{km}$ ) is given by:

(3) $r_{75}=$ effective size $/\left(\mathrm{M}_{75} * \mathrm{~S}\right)$

where effective size is $\mathrm{m}$, and $\mathrm{S}$ is the threshold angular triangle size (square-root area) of the thermal camera at the given thermal contrast in mrad $\left(\mathrm{S}^{-1}\right.$ is the TOD acuity in $\left.\mathrm{mrad}^{-1}\right)$. The probability vs range relationship with the TOD model is given by:

(4) $P(r)=1-4^{-\left(r_{75} / r\right)^{\beta}}=1-2^{-\left(r_{50} / r\right)^{\beta}}$

The recommended slope $\beta=1.25^{15}$. This results in a slope comparable to that with NVThermIP.

For this particular camera, the TOD sensor performance curve has already been measured ${ }^{26}$. At a given contrast, acuity can be determined by linear interpolation of the measured curves (Fig. 8 from Bijl et $\mathrm{al}^{26}$ ). The acuity per aspect angle (90L, 45L, 0, 45R, 90R) at corresponding thermal contrasts (see Table 1) is $0.50,0.52,0.52,0.55$ and $_{0.4} \mathrm{mrad}^{-1}$. The predicted $75 \%$ ID range based on the target size for the 5 angles (static conditions) are 10.2, 14.1, 16.1, 14.2 and 9.6 $\mathrm{m}$, 
respectively. For the average target contrast $(1.52 \mathrm{~K})$, we find an acuity of $0.50 \mathrm{mrad}^{-1}$ and given the average target size $(0.245 \mathrm{~m})$ predict the ID range to be $13.4 \mathrm{~m}$.

\section{RESULTS}

\subsection{Results 1: target orientation and motion}

The results of experiment 1 are shown in Figure 7 (static) and Figure 8 (static and dynamic). The left picture in Figure 7 shows the identification probability versus range for 5 orientations in the static condition with best fits, and the middle picture threat/non-threat discrimination. There is a clear effect of target orientation. In Figure 8 (left), 75\% correct ranges are plotted as a function of aspect angle (static: dark squares). Ranges differ over a factor of 3 ( 0.5 log units). The $75 \%$ correct range for threat/non-threat discrimination (31 m on average) was a constant factor of 2.2 larger than the $75 \%$ ID range.

The right-hand side picture in Figure 7 shows the average ID-scores for the static case over all orientations (filled circles), together with the best fit (black line). In addition, TOD and NVThermIP model predictions are given. Measured and predicted probability vs. range relationship are in close agreement: measured ID range $\left(\mathrm{r}_{75}=12.9 \mathrm{~m}\right)$ is within $6 \%$ from predictions for the TOD model $\left(\mathrm{r}_{75}=13.4 \mathrm{~m} ;+4 \%\right)$ and NVthermIP model $\left(\mathrm{r}_{75}=13.7 \mathrm{~m} ;+6 \%\right)$. The measured slope $(\beta=1.05)$ is somewhat shallower $(-15 \%)$ than assumed by the models $(\beta=1.25)$.

ID model predictions as a function of target orientation are plotted together with the experimental data in Figure 8 (left). The orientation effect for static data is nicely predicted by the NVThermIP and TOD models. The average difference between predicted and measured $r_{75}$ was less than $10 \%$ (-7\% for the NVThermIP model and $-9 \%$ for TOD model). Differences in curves for the TOD and NVThermIP are explained by a somewhat lower sensitivity to contrast in the TOD model.

In Figure 8 (left) also the $75 \%$ correct ranges for the dynamic imagery are plotted (open squares and diamonds). Unexpectedly, the results are remarkably similar to static performance: no significant difference was found (see also the Discussion).
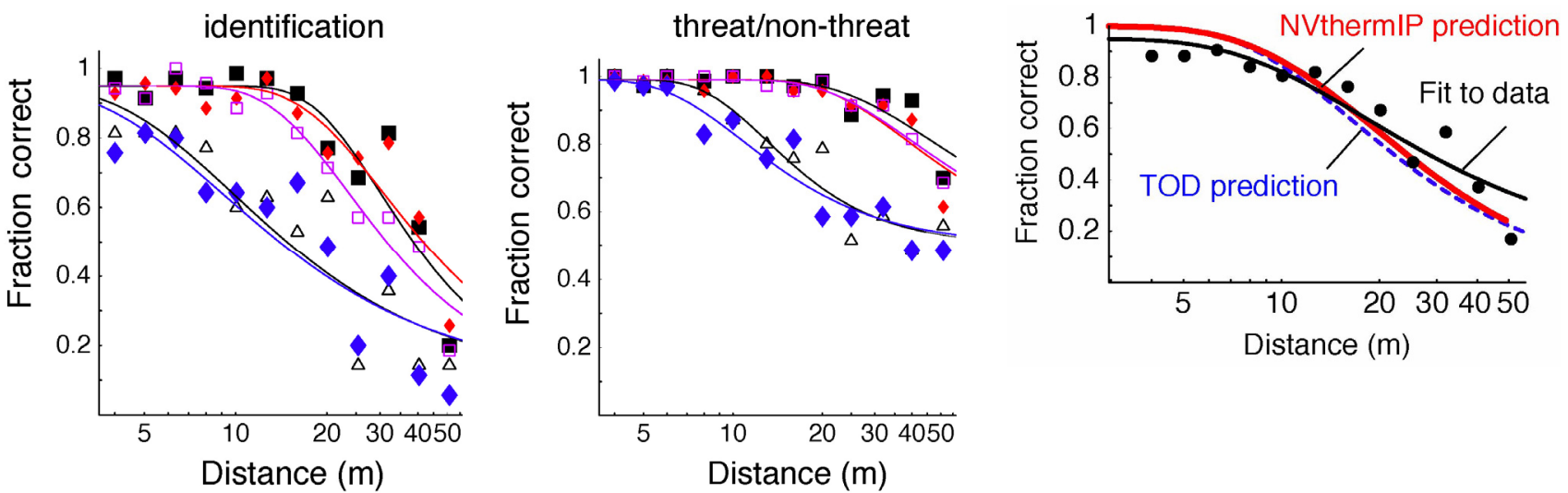

Figure 7. Results from experiment 1. Left: Identification probability versus range for 5 orientations in the static condition. The curves represent the best fits. Middle: Threat/non-threat discrimination versus range for 5 orientations in the static condition together with the best fits. Right: ID data averaged over all aspect angles. Aside from the best fit this graph contains the model predictions with the TOD and with NVThermIP. See text for details. 
Figure 8 (right) shows a confusion matrix from one of the experiments. The matrix shows that the responses cluster into the lower left quadrant (military weapons) or upper right quadrant (civil objects), except for the metal stick. This clustering indicates a high degree of similarity within two categories than over categories. The uniform responses to the stick likely reflects a high degree of similarity in form to both categories.
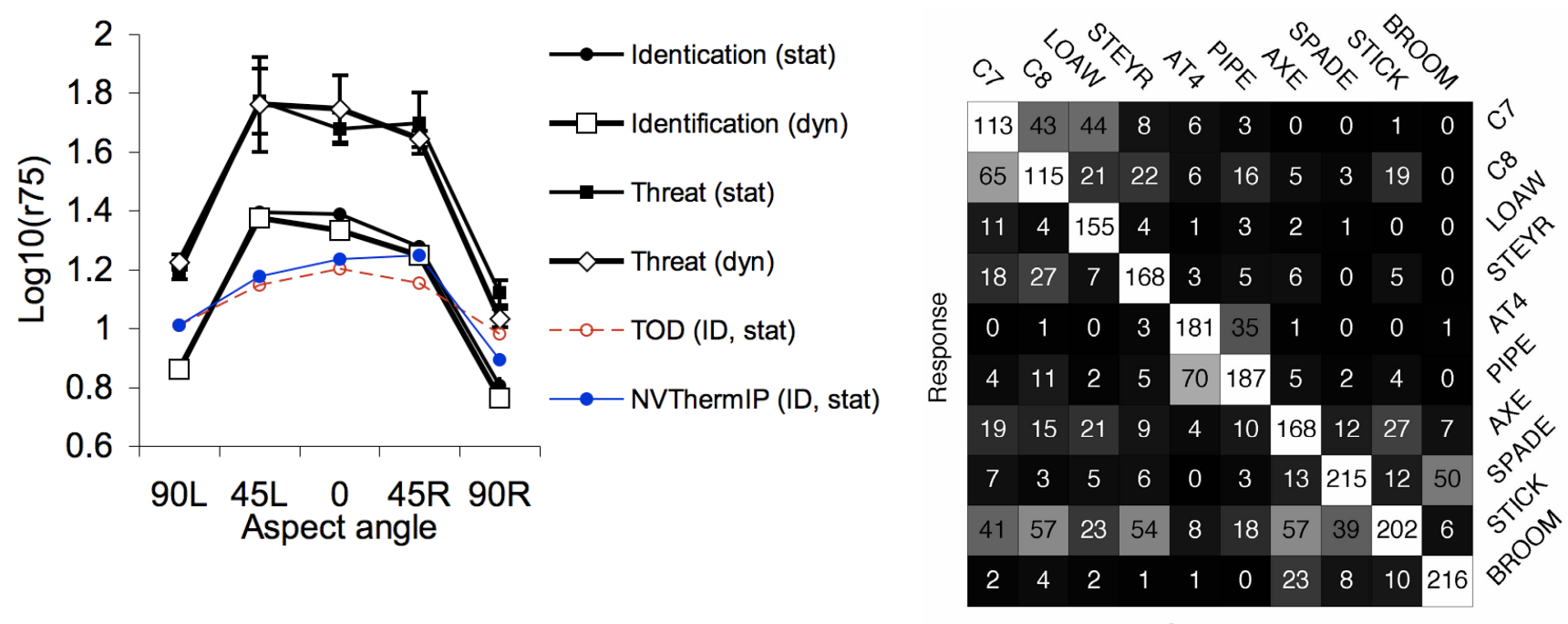

Figure 8: Left: Effect of aspect angle and motion (gray = static, dark = dynamic) on the $75 \%$ ID range (lower two curves). Also, threat/non-threat discrimination (upper two curves) are shown. For static, model predictions for identification are shown. Right: Confusion matrix for data at a range between 16 to $32 \mathrm{~m}$ on how often objects (horizontal position) was perceived to be another object (vertical position)

\subsection{Results 2: signal processing}

The effects of image processing and motion on the identification probability versus range relationship and 75\% ID ranges are shown in Figure 9 (left and right). Performance in the dynamic condition was clearly lower (more than two times the pooled standard deviation) than the static condition $(-16 \%)$, the reduction in $\mathrm{r}_{75}$ being 2.7 times larger than the pooled S.D.. We find no significant improvement (overlapping error bars) in the dynamic condition when playing back motion at a twice slower speed $(+6.5 \%)$, and no significant improvement (overlapping error bars) for a static sequence compared to a still image $(+9 \%)$.

LACE processing reduced the $75 \%$ ID range compared to static $(-12 \%)$ and dynamic $(-3.6 \%)$ conditions, but increased $\mathrm{r}_{75}$ compared to DSR4 (+2.5\%). On average, the effect $(-4.5 \%)$ was not significant.

Compared to the unprocessed dynamic condition, dynamic super resolution DSR 1 does not affect the $75 \%$ ID range significantly (+3\%), whereas DSR2 and DSR4 show a clear increase by $30 \%$ and $40 \%$, respectively.

Model predictions for the effect of motion and of DSR are given in Table 2. The effect of sensor motion on human performance is not yet modeled in NVThermIP. The effect of super-resolution is modeled, based on an optimum use of the additional sampling (Jacobs et al., 2005) ${ }^{11}$. The used orientation is $45^{\circ}(\mathrm{L}+\mathrm{R})$, which amounts to an average size of 0.255 and average dT of $2.02 \mathrm{~K}$.

According to Bijl et $\mathrm{al}^{26}$, sensor motion with the FLIR SC2000 results in a TOD increase of $25 \%$. DSR2 results in a range increase of $45 \%$, while DSR4 (average over $2 \mathrm{~K}$ and $20 \mathrm{~K}$ ) results in an increase of $52 \%$. As shown in Bijl et al already, NVThermIP predictions are in good agreement with these results. When comparing the predicted improvements by DSR with real measured ranges for static conditions, the measured range improvement for DSR4 
$(+17 \%)$ is much lower $(30 \%)$ than predicted $(+52 \%$ for DSR4). Note: the measured improvement for DSR relative to the dynamic condition $(+40 \%$ for DSR 4$)$ is only slightly $(10 \%)$ less than predicted $(+52 \%$ for DSR 4$)$.
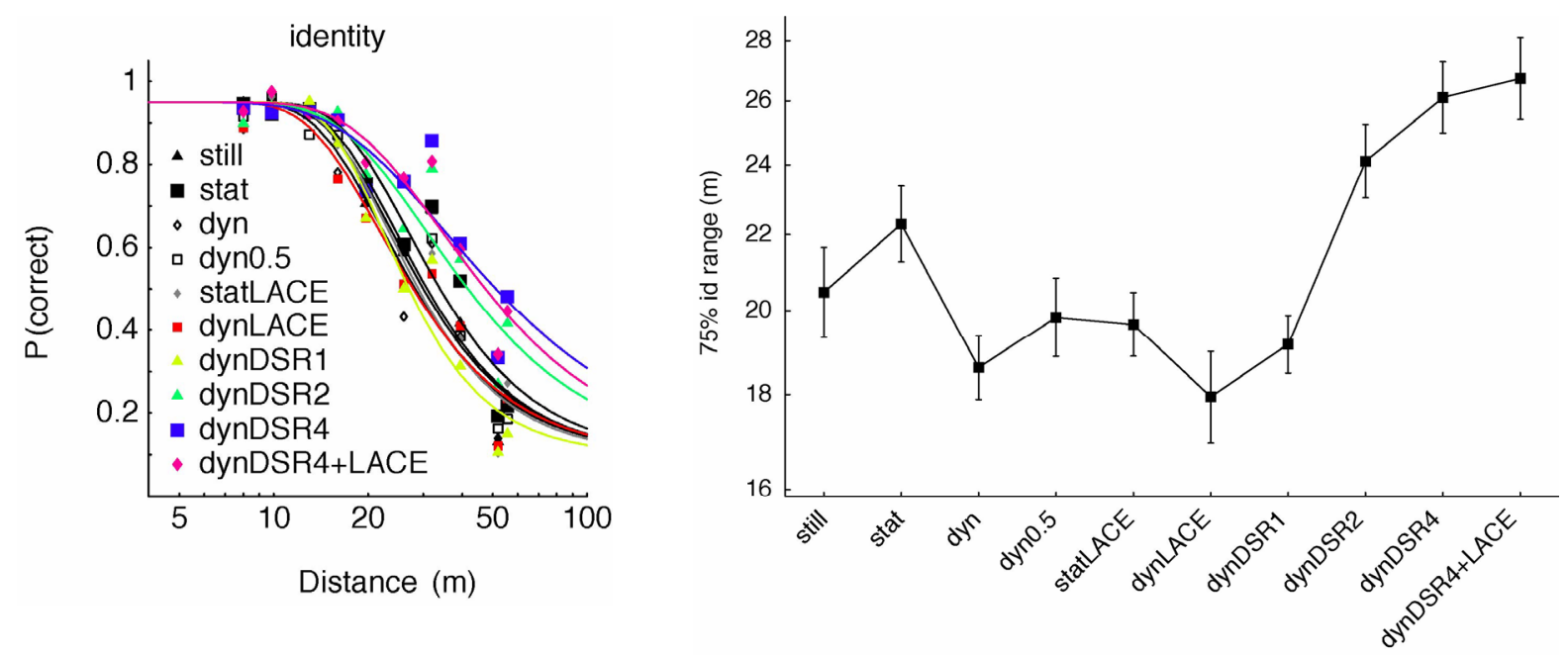

Figure 9. Left: Fraction of correct identification as a function of target distance for image processing conditions and fits. Each point represents the mean from 8 subjects (target aspect angle balanced over 45L and 45R). Right: Effect of image processing condition on the $75 \%$ ID range. Data are presented on a log scale with \pm 1 S.D. error bars derived from Monte-Carlo simulations.

Table 2: Measured and predicted 75\% ID ranges and/or relative increase compared to static (in parentheses)

\begin{tabular}{|l|l|l|l|}
\hline & NVThermIP prediction & TOD prediction & Measured \\
\hline static & $16.5 \mathrm{~m}$ & $14.1 \mathrm{~m}$ & $22.3 \mathrm{~m}$ \\
\hline dynamic & Not predicted & $(+25 \%)$ & $18.6 \mathrm{~m}(-16 \%)$ \\
\hline DSR2 & $23.5 \mathrm{~m}(+42 \%)$ & $(+45 \%)$ & $24.1 \mathrm{~m}(+8 \%)$ \\
\hline DSR4 & $26.0 \mathrm{~m}(+58 \%)$ & $(+52 \%)$ & $26.1 \mathrm{~m}(+17 \%)$ \\
\hline
\end{tabular}

\section{DISCUSSION}

Testing NVThermIP and TOD observer performance predictions with thermal recordings of two-handheld targets that are physically varied in distance, we find surprisingly small differences between predicted and measured identification ranges and slopes. The predictions are based on a scaling factor from Driggers et al $(2006)^{23}$, with data collected at only a single distance. These results suggest that, at least for the type of sensors under test, data range effects on sensor sampling by blurring images to (i.e. pixel rounding) as applied by the NVThermIP model are valid.

The effect of target orientation is quite well predicted with the current TA models such as the TOD model and NVThermIP based on the square-root area assumption for effective target dimension and the $\Delta$ TRSS measure for effective target thermal contrast. This is another indication that the square-root area assumption works pretty well even for very elongated targets (see also Bijl et al. ${ }^{35}$ for ship targets).

Contrary to the expectations, a small, negative effect of sensor motion on performance was found. In earlier studies with under-sampled imagers, considerable range increases were found for both abstract TOD test targets ${ }^{26,27,28}$ and real targets (Fanning et $\left.\mathrm{al}^{36}\right)$. A possible explanation is the higher speed used at recording ( 0.57 pixel/frame) compared to most previous studies (typically around $1 / 4$ pixel/frame). Higher speed increases motion smear in the image of an uncooled micro-bolometer camera and may also cause retinal smear if the eye track less well. Indeed, some motion smear was visible on individual images. Krapels ${ }^{25}$ used a similar speed but with a cooled MWIR camera that did not induce smear. We found little influence of playing back motion at lower speed, suggesting that motion smear on the 
recordings may have been the primary factor. Motion smear may also explain the reduced performance for the motionbased DSR2 and DSR4. If so, the relative high performance gain in motion conditions still found for DSR4 (40\%) compared to (50\% predicted) suggest that DSR is quite robust to motion blur.

LACE yields comparable results to non-LACE conditions that were optimized manually. These results imply that LACE can offer an improvement for all local features that is in the order of what can be reached by hand optimizing.

\section{CONCLUSIONS}

In an extensive observer performance experiment we determined the effect of target orientation, motion and image enhancement techniques for two hand-held targets on the identification and treat/non-threat discrimination performance with an uncooled LWIR micro-bolometer. We find the following:

- We found a strong effect of target orientation on performance: ID range varied over a factor of 3. The effect of target orientation is well predicted with the current TA models such as the TOD model and NVThermIP.

- The absolute identification range is very well predicted using the current models and the recommended criteria. In this study, we find that threat/non-threat discrimination ranges for two hand-held targets are about two times longer than identification ranges.

- Contrary to the expectations, no effect of sensor motion on performance was found. Similarly, the effect of DSR was less strong than expected or predicted by the models. The difference is probably due to smear induced in the micro-bolometer detectors because of a higher motion speed used in the present experiment.

- Performance with LACE was similar to performance on an image optimized by hand.

\section{REFERENCES}

1. Ratches, J.A. (1976). Static Performance Model for Thermal Imaging Systems. Optical Engineering, 15, 6, 525530, 1976.

2. Valeton, J.M. \& Bijl, P. (1994). Target aquisition: human observer performance studies and TARGAC model validation (Report TNO-TM 1994 B-15). Soesterberg, The Netherlands: TNO Human Factors Research Institute.

3. Bijl, P., Valeton, J.M. \& de Jong, A.N. (2000). TOD predicts target acquisition performance for staring and scanning thermal imagers, SPIE Proceeding Vol. 4030, 96-103.

4. Driggers, R.G., Wittenstein, W., Williams, W., Bijl, P., Lopez, J.M. (2003). Modeling of Undersampled Imaging Systems. NATO Technical Report RTO-TR-075(I), AC/323(SET-015)TP/25.

5. Ratches, J.A., Vollmerhausen, R.H., Jacobs, E., \& Driggers, R.G. (2001)Target acquisition performance modeling of infrared imaging systems: past, present, and future," IEEE Sensors Journal 1, 31-40 (2001).

6. Wittenstein, W., Fick, W. \& Raidt, U. (1996). Range Performance of Two Staring Imagers - Presentation of the Field Trial and Data Analysis. Proc. SPIE Conf. on Infrared Imaging Systems, 2743, 132, (1996).

7. K. Krapels, R. G. Driggers, E. Jacobs, S. Burks, and S. Young, "Characteristics of infrared imaging systems that benefit from superresolution reconstruction," Appl. Opt. 46, 4594-4603 (2007)

8. Holst, G.C. (2007) Imaging system performance based upon F /d. Optical Engineering 46, Issue 10, 103204

9. Driggers, R.G., Vollmerhausen, R., Wittenstein, W., Bijl, P., Valeton, J.M. (2000). Infrared Imager Models for Undersampled Imaging Systems. Proc. Fourth Joint International Military Sensing Symposium, 45, 1, $335-346$.

10. Vollmerhausen, R., \& Driggers, R.G (1999). NVTherm: next generation night vision model. Proc. IRIS Passive Sensors, 1, 121-134.

11. Jacobs, E. \& Driggers, R. G. (2005). NVThermIP modeling of super-resolution algorithms. Proc. SPIE, Vol. 5784$19,125-135$.

12. Wittenstein, W. (1999). Minimum temperature difference perceived - a new approach to assess undersampled thermal imagers. Optical Engineering 38, 5, 773 - 781.

13. Bijl, P.\& Valeton, J.M. (1998). TOD, the alternative to MRTD and MRC. Optical Engineering 37, 7, 1976 - 1983. 
14. Hogervorst, M.A., Bijl, P. \& Valeton, J.M. (2001). Capturing the sampling effects: a TOD sensor performance model. Proc. SPIE, Vol. 4372, 62-73.

15. Bijl, P. \& Hogervorst, M.A. (2007). NVThermIP vs TOD: matching the Target Acquisition range criteria. SPIE Proc. SPIE, Vol. 6543, pp. 65430C.

16. Bijl, P., Hogervorst, M.A. \& Valeton, J.M. (2002). TOD, NVTherm and TRM3 model calculations: a comparison. Proc. SPIE, Vol. 4719, 51-62.

17. S. Moyer, J. G. Hixson, T. C. Edwards, and K. Krapels, "Probability of identification of small hand-held objects for electro-optic forward-looking infrared systems," Opt. Eng. 45, 063201 (2006).

18. S. K. Moyer, E. Flug, T. C. Edwards, K. A. Krapels, and J. Scarbrough, "Identification of handheld objects for electro-optic FLIR applications," Proc. SPIE, Vol. 5407, 116-126 (2004).

19. S. Moyer and N. Devitt, "Resolvable cycle criteria for identifying personnel based on clothing and armament variations," Proc. SPIE, Vol. 5784, 60-71 (2005).

20. N. Devitt, J. G. Hixson, S. Moyer, and E. Flug, "Urban vehicle cycle criteria for identification," Proc. SPIE, Vol. 5784, 48-59 (2005).

21. K. Krapels, R. G. Driggers, D. Deaver, S. K. Moker, and J. Palmer, "Midwave infrared and visible sensor performance modeling: small craft identification discrimination criteria for maritime security," Appl. Opt. 46, 73457353 (2007).

22. Driggers R. G., Larsen, L., Fanning, J., Hixson, J. G. \& Krapels, K. (2007). Direct View Optics Model For Facial Identification. Proc. SPIE, Vol. 6543.

23. Driggers, R. G., Jacobs, E. L., Vollmerhausen, R. H., O'Kane, B. L., Self, M., Moyer, S., Hixson, J. G., Page, G., Krapels, K., Dixon, D., Kistner, R. \& Mazz, J. (2006). Current infrared target acquisition approach for military sensor design and wargaming. Proc. SPIE, Vol. 6207, 94-110.

24. Vollmerhausen, R. H., Driggers, R. G., O’Kane, B. L. (1999). Influence of sampling on target recognition and identification," Opt. Eng., 38, 763.

25. Krapels, K., Driggers, R.G. \& Teaney, B. (2005). Target-acquisition performance in under-sampled infrared imagers: static imagery to motion video. Applied Optics, 44 (33), 7055-7061.

26. Bijl, P., Schutte, K. \& Hogervorst, M.A. (2006). Applicability of TOD, MRT, DMRT and MTDP for dynamic image enhancement techniques. Proc. SPIE, Vol. 6207

27. Driggers, R.G., Krapels, K., Murrill, S., Young, S.S., Thielke, M. \& Schuler, J. (2004). Superresolution performance for undersampled imagers. Optical Engineering 44, 1.

28. Jonathan Fanning, Justin Miller, Jennifer Park, Gene Tener, Joseph Reynolds, Patrick O’Shea, Carl Halford, Ron Driggers (2007). IR system field performance with super-resolution. Proc. SPIE, Vol. 6543.

29. Young, S.S. \& Driggers, R. G. (2005). Super-resolution image reconstruction from a sequence of aliased imagery. Proc. SPIE, Vol. 5784-18, 51-62.

30. Park, S.C., Park, M.K. \& Kang, M.G. (2003). Super-resolution image reconstruction: a technical overview. IEEE Signal Processing Magazine, 21-36.

31. Schutte, K, de Lange, D.J., van den Broek, S.P., (2003). Signal conditioning algorithms for enhanced tactical sensor imagery, Proc. SPIE, Vol. 5076, 92-100.

32. Driggers et al. (2006). Current infrared target acquisition approach for military sensor design and wargaming Infrared imaging systems. Proc. SPIE, Vol. 6207, pp. 620709.1-620709.17

33. Snoeren, P.R., Puts M.J.H. (1997). Multiple Parameter Estimation in an adaptive psychometric method: Muest, an extension of the Quest method, Journal of Mathematical Psychology, 41, 431-439.

34. Schutte, K. (1997) Multi-scale adaptive gain control of IR images. Infrared technology and applications XXIII; Proceedings of the Conference, Orlando, FL; UNITED STATES; 20-25 Apr. 1997. pp. 906-914. 1997"

35. Bijl, P.\& Valeton, J.M. (1998). Validation of the new TOD method and ACQUIRE model predictions using observer performance data for ship targets. Optical Engineering 37, 7, 1984 - 1994.

36. Fanning, J. et al. (2007). IR system field performance with superresolution. Proc. SPIE 6543. 